\title{
Rapid prototyping of advanced control schemes in ASDEX Upgrade
}

\author{
B.Sieglin ${ }^{\mathrm{a}}$, M.Maraschek ${ }^{\mathrm{a}}$, O.Kudlacek ${ }^{\mathrm{a}}$, A.Gude $^{\mathrm{a}}$, W.Treutterer ${ }^{\mathrm{a}}$, M.Kölbl $^{\mathrm{a}}$, A.Lenz ${ }^{\mathrm{a}}$, the ASDEX Upgrade Team ${ }^{\mathrm{a}}$, \\ the EUROfusion MST1 Team ${ }^{\mathrm{b}}$
}

${ }^{a}$ Max-Planck-Institute for Plasma Physics, Boltzmannstr. 2, D-85748 Garching

${ }^{b}$ see B. Labit et al 2019 Nucl. Fusion 59086020

\begin{abstract}
The integration of advanced control schemes is becoming more important as the development of fusion experiments progresses. The ASDEX Upgrade discharge control system (DCS) is designed to be adaptable via configuration, no recompilation is necessary to tailor the behaviour of the control system.

In order to enable advanced control schemes the required information has to be available during the discharge. With the DCS satellite concept the control system can easily be extended by including e.g. diagnostics, actuators and data processing nodes. In this paper the extension of DCS for rapid prototyping of control schemes is discussed.

To easily add and modify input signals used for advanced control schemes, without the requirement to have expert knowledge of the control system by the experimentalist, a new application process (AP) has been implemented using the $\mathrm{C}++$ Mathematical Expression Toolkit Library (ExprTk), which enables the inclusion of run-time mathematical expressions into DCS. The AP is operated separately from the central DCS as a DCS satellite. This is done to be able to test and validate the calculations without having to make changes to DCS.

For tokamak operation, disruptions pose a major threat especially for large devices such as ITER. Therefore, disruption avoidance is an active field of research and the inclusion of avoidance schemes into DCS as part of exception handling is ongoing. For the case of H-Mode density limit disruptions an avoidance scheme using central heating has been implemented and successfully tested on ASDEX Upgrade, exploiting these new features for rapid development and testing.
\end{abstract}

\section{Introduction}

The integration of advanced control schemes is becoming more important as the development of fusion experiments progresses. The ASDEX Upgrade discharge control system [1, 2] (DCS) is designed to be adaptable via configuration, no recompilation is necessary to tailor the behaviour of the control system.

In order to enable advanced control schemes the required information has to be available during the discharge. With the DCS satellite concept [3] the control system can easily be extended by including e.g. diagnostics, actuators and data processing nodes.

To enable a quick and easy inclusion of new signals into DCS for the purpose of control, the DCS capabilities have been extended using the $\mathrm{C}++$ Mathematical Expression Toolkit Library (ExprTk) [4]. With this it is now possible to use existing signals and process them to calculate new signals (e.g. combined quantities, scalings, etc) for control or as input for further calculations. All this is possible by configuration only, keeping the DCS philosophy to be able to change the behaviour without having to recompile the control system.

The aim of this extension is to allow rapid prototyping of advanced control schemes in ASDEX Upgrade. Depending on the desired control scheme not all quantities needed for control are readily available. This is for example the case for derived quantities or empirical scalings.

This DCS extension has been successfully tested for the disruption avoidance scheme for H-Mode density limit disruptions [5], where an empirical disruption predictor is used to control the amount of central auxiliary heating.

\section{Desired Workflow}

By nature DCS is a complex system requiring expert knowledge for its operation and configuration. The experimentalist, however, does not necessarily have this knowledge, but still might want to include his own signals for control. The desired workflow (Figure 1) should enable everyone to define the calculation rule for a signal, which is then converted to the DCS configuration and instantiated as needed.

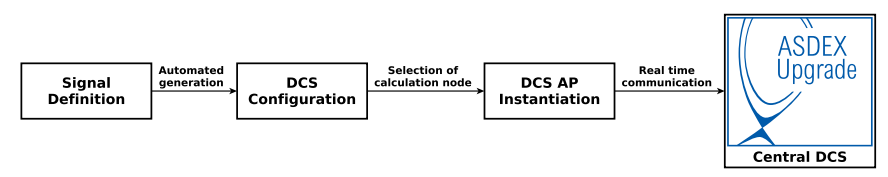

Figure 1: Desired workflow for rapid prototyping of real time signals into the ASDEX Upgrade control system. 
Table 1: Example YAML file for the definition of the Greenwald density.

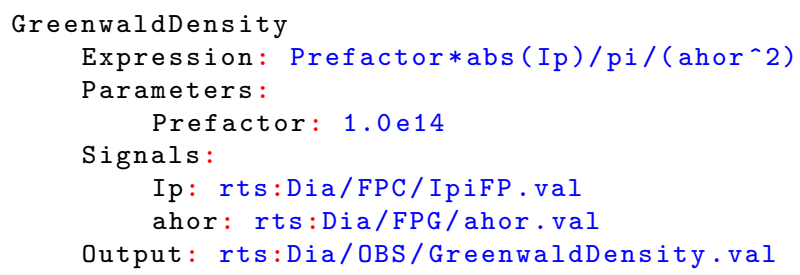

For the definition of the calculation rule YAML Ain't Markup Language (YAML) was chosen, due to its clear and descriptive nature.

The YAML configuration contains the expression which will be evaluated in each cycle during the real time phase. Parameters are values which can be used with their name within the expression. The signals are identified via their name in the expression and their configuration value identifies the real time signal to be used. The output specified the DCS real time signal name under which the result is available during the real time phase. As an example for such a configuration the signal definition of the Greenwald density, the empirical maximum achievable electron density for tokamaks, is shown in table 1.

Each signal is processed in a dedicated thread during the runtime phase. From the YAML configuration the DCS configuration is generated which is used to configure DCS for the runtime phase. The decision where the signal processing is done is taken by the DCS operator. The DCS satellite concept enables to distribute the calculation load onto multiple calculation nodes. The task scheduling is done automatically by DCS using the signal dependencies given in the signal definition.

\section{Implementation into DCS}

The ASDEX Upgrade DCS is implemented in $\mathrm{C}++$ and uses so called application processes (AP) as building blocks for the realization of the required functionality needed for the control of ASDEX Upgrade. During runtime each instance of an AP runs in its own thread allowing for concurrent execution of multiple APs within one process. For the inclusion of the ability to compute signals which are defined via configuration, an AP has been implemented which encapsulates the capabilities of ExprTk. The current implementation of the AP allows the calculation of one scalar output signal, taking multiple scalar input signals and parameters. So far the AP has only been used for floating point signals, but can be adapted to accept other signal types (e.g. integers) due to the templated nature of the ExprTk. The output signal value of the previous time point can be accessed in the expression using the reserved variable name result. With this recursions with the previous computation result are possible.

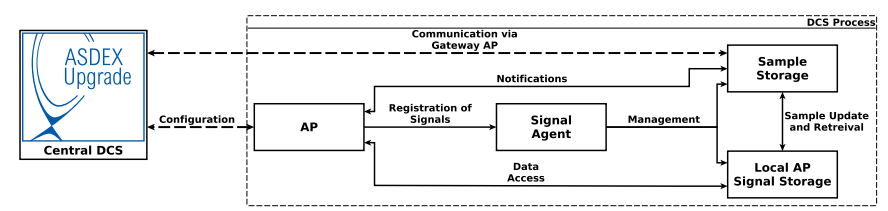

Figure 2: Schematic illustration of the signal storage and communication mechanism within the ASDEX Upgrade DCS.

The internal mechanism for signal storage and communication within DCS is shown in figure 2. When a discharge is loaded all produced and consumed signals and parameters are registered with the signal agent. For each AP the required storage is locally allocated. In addition the required sample storage, which can contain the history of the signal, is allocated globally for each signal.

The references to the respective local signals and parameters are handed to ExprTk which parses the expression and builds the abstract syntax tree (AST) connecting the signals and parameters with the desired calculation. The result is an ExprTk kernel which can be evaluated. When the kernel is evaluated the current values in the local signal storage are used and the result is stored in the reference to the local output signal.

During the real time phase the memory position of the signals is fixed for each AP. The communication with other DCS satellites and central DCS is performed using a gateway AP. This gateway AP manages sending and receiving of samples. Once a new data sample is received and stored inside the sample storage, a notification is sent to all APs requesting this signal. The AP then retrieves the new sample and stores it in its local storage. The ExprTk kernel is then evaluated, after which the result of the evaluation is available in the local signal storage. The AP then notifies the sample storage that the new sample of the output signal is available and this is then copied to the sample storage where it is made available to other APs, including the gateway AP which distributes the sample to other DCS satellites and central DCS.

After each evaluation of the ExprTk kernel it is checked if the result is a valid number. If this is not the case the sample is tagged as invalid using the standard DCS capabilities [1]. Each consumer of the signal can thus check if the signal can be used.

\section{Application to disruption avoidance}

Disruptions in tokamak fusion devices are a severe issue, resulting in a sudden uncontrolled termination of the plasma, which poses the threat of machine damage. As one line of defence before a disruption cannot be avoided anymore and disruption mitigation $[6,7]$ has to be conducted, disruption avoidance aims at keeping the plasma within a controllable state at all times.

One type of disruptions occurs in high confinement $(\mathrm{H}-$ mode) discharges with high density, the so called H-mode density limit disruptions. 
For the disruption avoidance scheme the calculated signals, namely the confinement scaling $H_{98, y 2}$ and the empirical critical edge density fraction $n_{\mathrm{e}, \text { edge }} / n_{\mathrm{e}, \mathrm{edge}, \mathrm{crit}}$ are used. Where the critical edge density is taken from [8] as

$n_{\text {e,edge,crit }}\left[\mathrm{m}^{-3}\right]=5 \cdot 10^{19} \cdot P_{\text {tot }}[\mathrm{MW}]^{0.396} \cdot I_{\mathrm{p}}[\mathrm{MA}]^{0.265} \cdot q_{95}^{-0.323}$

and the edge density $n_{\mathrm{e}, \text { edge }}$ is taken from the edge channel of the ASDEX Upgrade DCN interferometer [9].

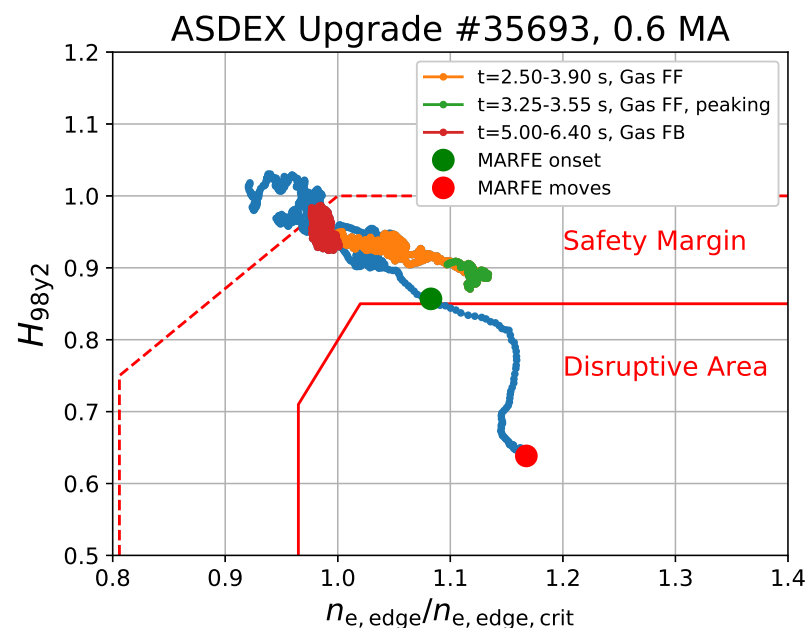

Figure 3: Overview of ASDEX Upgrade discharge \#35693 with active H-mode limit disruption avoidance controller. Note: The shown safety margin is shown only for illustration.

These two parameters span a state space in which an empirical area of disruptivity is defined which the scheme tries to avoid. The area of disruptivity is indicated as the red box in figure 3 . In case the discharge approaches this area central auxiliary heating is requested from actuator management $[10,11]$. The heating is continuously increased with decreasing distance up to full allocated heating power when the border is reached.

For this kind of scenario it was found that the application of central heating could avoid a disruption. For the test of the control scheme central electron cyclotron resonance heating $(\mathrm{ECRH})$ was controlled. A power request is calculated utilizing the DCS capabilities described above. The power request is issued to actuator management which then modulates the gyrotrons to achieve the desired heating power.

Figure 4 shows the signal hierarchy for the density limit disruption avoidance experiments. The green boxes indicate signals that were already produced by diagnostics and available as input signals. The blue boxes show the signals that were implemented using the rapid prototyping. Each signal calculation runs in its own DCS AP instance, with a dedicated thread. In each DCS cycle the AP waits until all input signals needed for the calculation are available. Once this is the case the configured expression is evaluated and the result is published using the standard DCS signal distribution mechanisms. The benefit from this is that the

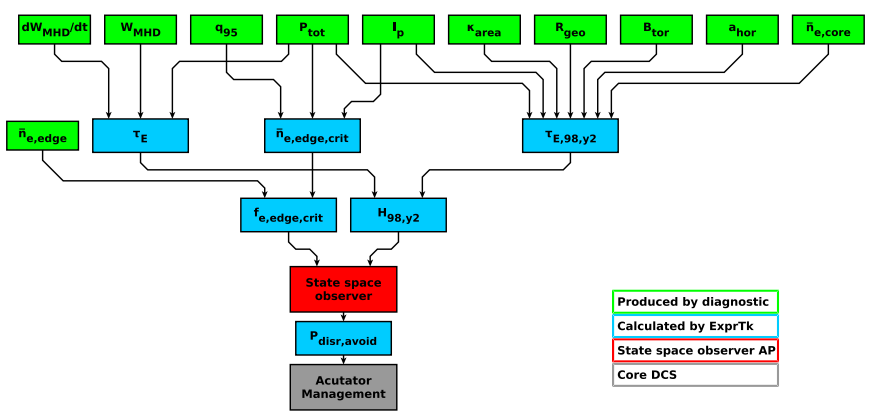

Figure 4: Signal hierarchy for H-mode density limit disruption avoidance.

scheduling when the different signals are calculated within a DCS cycle is automatically done by DCS. In figure 4 this results in an information flow in each cycle from top to bottom, it starts with the diagnostic signals and ends at the actuator management (grey box) which issues the command to the heating sources.

For testing of the control scheme the decision logic has been bypassed and the control scheme has been activated in a preprogrammed fashion during a discharge which, without interference, would encounter a density limit disruption. For the power request a linear increase with decreasing distance is chosen. The power request starts at zero at a distance of 0.15 from the boundary linearly increasing to full power up to zero distance where the boundary is hit. The distance of 0.15 was chosen empirically and symmetric for both axis since both $H_{98, y 2}$ and $n_{\mathrm{e}, \text { edge }} / n_{\mathrm{e}, \text { edge,crit }}$ are around unity for these experiments. In turn the gyrotrons are modulated to deliver the requested power.

An example of a successful application of this disruption avoidance scheme with active controller is shown in figure 5. In the first plot the plasma current $I_{\mathrm{p}}$ and the plasma stored energy $W_{\text {MHD }}$ are shown. The second plot contains the Greenwald density $n_{\mathrm{GW}}$ and the central and edge channel of the DCN interferometer [9] providing the line average electron density. The two critical quantities for the disruption avoidance controller $H_{98, \text { y2 }}$ and the normalized critical edge density $f_{\mathrm{e}, \text { edge,crit }}=$ $n_{\mathrm{e}, \text { edge }} / n_{\mathrm{e} \text {,edge,crit }}$ are presented in the third plot. The normalized power request determined by the disruption avoidance controller $P_{\text {disr,avoid }}$ is in the fourth plot. In the fifth plot the injected auxiliary heating by both ECRH and Neutral Beam Injection (NBI) are shown. In the last plot the deuterium gas fuelling request is shown.

The discharge was split into three main phases. In the first phase from 2.5 to 4 seconds the density is increased by a feed forward deuterium gas puff with a rate of $5.73 \cdot 10^{22} \mathrm{e} / \mathrm{s}$, which was the disruptive limit found in a disruptive reference discharge. The application of central heating is able to stabilize the discharge, peaking at a density of 1.1 times the Greenwald density. In the second phase from 4 to around 6.5 seconds a density ramp is applied. At the lowest density the controller does not 


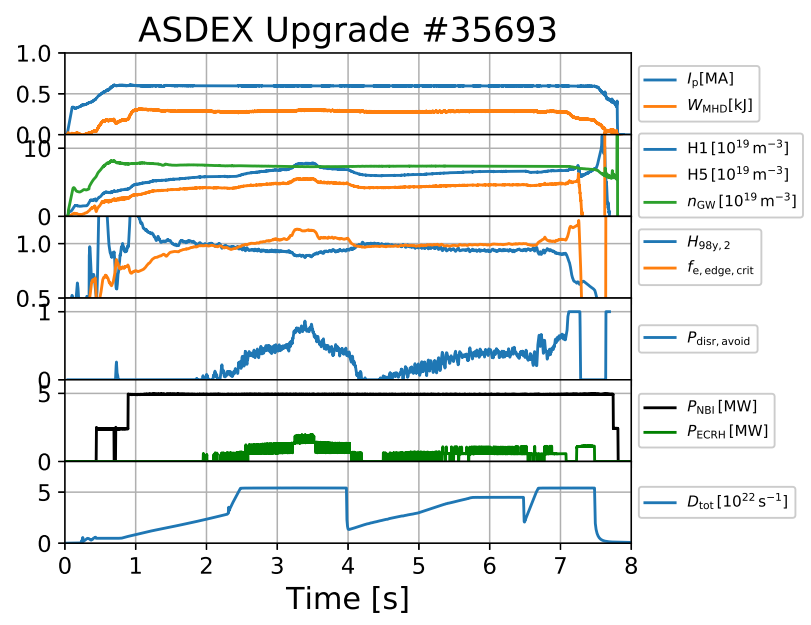

Figure 5: Overview of ASDEX Upgrade discharge \#35693 with active H-mode limit disruption avoidance controller.

request any additional heating power. With increasing density the controller starts to request heating power and stabilizes the discharge. In the third phase the gas puff is switched back to feed forward with the level of the first phase, with active controller. The discharge is kept stable away from the disruptive area. To study the MARFE [12] behaviour the disruption avoidance controller is switched off at the end of the third phase. The additional ECRH heating is ramped down in feed forward, switched off and after $130 \mathrm{~ms}$ switched back on again with two sources. After the switch off of the gyrotrons the MARFE forms and starts moving away from the $\mathrm{X}$-point region. The application of the two gyrotrons after the $130 \mathrm{~ms}$, during which the MARFE has evolved, is not sufficient to stabilize the discharge, which in consequence disrupts.

Figure 3 shows the evolution of the discharge in the state space diagram used for the disruption avoidance scheme. It is observed that the applied additional heating power is keeping the discharge away from the boundary. After the avoidance scheme is deactivated and the end of the third phase of the discharge is reached, the discharge enters the disruptive area. There the MARFE has its onset. As the discharge continues to lose confinement it moves further into the disruptive area where the MARFE starts to move away from the $\mathrm{X}$-point region towards the plasma top. At this point the density measurement from the interferometry is corrupted by the strong localised density increase due to the MARFE and the discharge disrupts.

This shows that the active control prevented the formation of a MARFE at the X-point. After the MARFE has developed and moved away from the X-point the application of central ECRH, even with higher heating power compared to the controlled phase, is not sufficient to stabilize the discharge and prevent the disruption.

\section{Conclusions}

The capability to include runtime calculated signals via configuration has been implemented into the ASDEX Upgrade DCS. The implementation, using the ExprTk, has proven to be sufficient for the low latency requirements of DCS. The configuration utilizes YAML for ease of use for the experimentalist who does not require expert knowledge about the underlying system.

The ability for rapid prototyping of advanced control schemes has been demonstrated for H-mode density limit disruption avoidance. The required signals have been calculated using the new runtime capabilities and the results have been used as input for a controller, which in turn issues a heating power request to actuator management. Using ECRH the control scheme is able to stabilize the plasma and avoid the density limit disruption. This shows that an early action on the deviation of the plasma state from the nominal path is useful for disruption avoidance reducing the required actuator usage.

This control scheme proved to stabilize the discharge with minimal intrusion and is now available to the experimentalist by default. It is foreseen to utilize the rapid prototyping capabilities to further control schemes to decrease development time and increase the operational capabilities of ASDEX Upgrade. However, the capabilities of this system are not limited to rapid prototyping but can also be applied to similar tasks, e.g. signal generation or general signal processing.

\section{Acknowledgement}

This work has been carried out within the framework of the EUROfusion Consortium and has received funding from the Euratom research and training programme 2014 - 2018 and 2019 - 2020 under grant agreement No 633053. The views and opinions expressed herein do not necessarily reflect those of the European Commission.

\section{References}

[1] W. Treutterer, R. Cole, K. Lüddecke, G. Neu, C. Rapson, G. Raupp, D. Zasche, and T. Zehetbauer. ASDEX Upgrade Discharge Control System-A real-time plasma control framework. Fusion Engineering and Design, 89(3):146 - 154, 2014. Design and implementation of real-time systems for magnetic confined fusion devices.

[2] Wolfgang Treutterer, Richard Cole, Alexander Gräter, Klaus Lüddecke, Gregor Neu, Christopher Rapson, Gerhard Raupp, Dieter Zasche, and Thomas Zehetbauer. Transforming the ASDEX Upgrade discharge control system to a general-purpose plasma control platform. Fusion Engineering and Design, 9697:712 - 715, 2015. Proceedings of the 28th Symposium On Fusion Technology (SOFT-28).

[3] B. Sieglin, W. Treutterer, and L. Giannone. Dcs satellite: Enhanced plant system integration on asdex upgrade. Fusion Engineering and Design, 146:1737 - 1740, 2019. SI:SOFT-30.

[4] $\mathrm{C}++$ mathematical expression library. http://www.partow. net/programming/exprtk/. 
[5] M Maraschek, A Gude, V Igochine, H Zohm, E Alessi, M Bernert, C Cianfarani, S Coda, B Duval, B Esposito, S Fietz, M Fontana, C Galperti, L Giannone, T Goodman, G Granucci, L Marelli, S Novak, R Paccagnella, G Pautasso, P Piovesan, L Porte, S Potzel, C Rapson, M Reich, O Sauter, U Sheikh, C Sozzi, G Spizzo, J Stober, W Treutterer, and and. Pathoriented early reaction to approaching disruptions in ASDEX Upgrade and TCV in view of the future needs for ITER and DEMO. Plasma Physics and Controlled Fusion, 60(1):014047, nov 2017.

[6] M. Lehnen, K. Aleynikova, P.B. Aleynikov, D.J. Campbell, P. Drewelow, N.W. Eidietis, Yu. Gasparyan, R.S. Granetz, Y. Gribov, N. Hartmann, E.M. Hollmann, V.A. Izzo, S. Jachmich, S.-H. Kim, M. Kočan, H.R. Koslowski, D. Kovalenko, U. Kruezi, A. Loarte, S. Maruyama, G.F. Matthews, P.B. Parks, G. Pautasso, R.A. Pitts, C. Reux, V. Riccardo, R. Roccella, J.A. Snipes, A.J. Thornton, and P.C. de Vries. Disruptions in ITER and strategies for their control and mitigation. Journal of Nuclear Materials, 463:39 - 48, 2015. PLASMA-SURFACE INTERACTIONS 21.

[7] G Pautasso, M Bernert, M Dibon, B Duval, R Dux, E Fable, J C Fuchs, G D Conway, L Giannone, A Gude, A Herrmann, M Hoelzl, P J McCarthy, A Mlynek, M Maraschek, E Nardon, G Papp, S Potzel, C Rapson, B Sieglin, W Suttrop, W Treutterer, and and. Disruption mitigation by injection of small quantities of noble gas in ASDEX Upgrade. 59(1):014046, nov 2016.

[8] M Bernert, T Eich, A Kallenbach, D Carralero, A Huber, P T Lang, S Potzel, F Reimold, J Schweinzer, E Viezzer, and $\mathrm{H}$ Zohm. The H-mode density limit in the full tungsten ASDEX Upgrade tokamak. Plasma Physics and Controlled Fusion, 57(1):014038, nov 2014.

[9] A. Mlynek, L. Casali, O. Ford, and H. Eixenberger. Fringe jump analysis and implementation of polarimetry on the ASDEX Upgrade DCN interferometer. Review of Scientific Instruments, 85(11):11D408, 2014.

[10] O. Kudlacek, W. Treutterer, F. Janky, B. Sieglin, and M. Maraschek. Actuator management development on ASDEXUpgrade. Fusion Engineering and Design, 2019.

[11] O. Kudlacek, W. Treutterer, B. Sieglin, F. Janky, F. Felici, I. Gomez-Ortiz, A. Gräter, T. Maceina, M. Maraschek, T. Zehetbauer, the ASDEX Upgrade Team, and The EUROFusion MST1 Team. Use of virtual actuators in ASDEX Upgrade Control. Fusion Engineering and Design. This conference.

[12] Vladislav Kotov, D. Reiter, and S. Wiesen. Self-consistent modeling of X-point MARFE and divertor detachment. Journal of Nuclear Materials, 438:S449 - S452, 2013. Proceedings of the 20th International Conference on Plasma-Surface Interactions in Controlled Fusion Devices. 